\title{
Wo Mann sich traut - Heiratsentscheidungen westdeutscher Männer im räumlichen Kontext
}

\author{
Von Karsten Hank ${ }^{1}$
}

\section{Einleitung}

Vor inzwischen zehn Jahren stellte Hans Bertram in einem Interview fest, »daß nur durch die Berücksichtigung kultureller und sozialräumlicher Faktoren ein angemessenes Bild der Lebenssituation von [...] Familien zu entwickeln ist.« (Bertram 1993, S.37) Seither sind eine Reihe zum Teil sehr elaborierter, jedoch weitgehend deskriptiver Studien regionaler Familien- und Haushaltsstrukturen in Deutschland entstanden (z.B. Bertram/Nauck/Klein 2000; Nauck/Bertram 1995). Es ist jedoch von Nauck (1995, S.119) zurecht darauf hingewiesen worden, dass »[e]ine wie immer verfeinerte Regionaltypologie [...] lediglich ein erster Schritt für das weitergehende Ziel sein [kann], die Basis für empirische Mehrebenen-Analysen zu legen, bei der Aggregat- und Individual-Daten für eine kontextualisierte Erklärung familiären Verhaltens miteinander verknüpft werden.«

Entsprechende Analysen des Einflusses regionaler sozialer Kontexte auf die Familiengründung westdeutscher Frauen in den 1980er und 1990er Jahren unterstreichen die Notwendigkeit und Fruchtbarkeit eines solchen Ansatzes (Hank 2002, 2003). Die wichtigsten Befunde dieser Untersuchungen auf Basis des Sozio-oekonomischen Panels sind, dass regionale Unterschiede im Geburtenverhalten weitestgehend durch Kontrolle individueller Merkmale - vor allem Familienstand und Alter der Frau - erklärt werden können (vgl. auch Kopp 2000), während die regionale Varianz im Heiratsverhalten weder auf Bevölkerungskompositionseffekte noch auf strukturelle Kontexteffekte zurückgeführt werden kann. Hieraus wird auf die Existenz raumgebundener sozio-kultureller Milieus geschlossen, die sich z.B. in der Akzeptanz nicht-ehelicher Lebensgemeinschaften oder in kollektiven Erwartungen hinsichtlich des Heiratsalters voneinander unterscheiden (vgl. Hank 2003).

Hieran anschließend untersucht die vorliegende Studie den Zusammenhang zwischen Merkmalen des räumlichen Kontexts, der auf der Ebene von Stadt- und Landkreisen definiert wird, und dem Übergang zur ersten Ehe bei westdeutschen Männern in den 1980er und 1990er Jahren. Dies ist insofern bedeutsam, als Klein (2003, S.524) zeigen konnte, dass die »Zunehmende Kinderlosigkeit im Lebenslauf [...] aus der erhöhten Instabilität von Partnerschaften und bei Männern zusätzlich aus dem Männerüberschuss in der jüngeren Bevölkerung « resultiert. Abbildung 1 zeigt die räumliche Verteilung der rohen Heiratsziffern in den westdeutschen Kreisen für den entsprechenden Zeitraum. ${ }^{2}$ Ziel der Untersuchung ist es, herauszufinden, ob sich die Befunde der oben zitierten Analyse des Heiratsverhaltens von Frauen für Männer replizieren lassen, bzw. inwieweit geschlechtsspezifische Kontexteinflüsse auf die individuelle Entscheidung zur Eheschließung festgestellt werden können (vgl. Lloyd/ South 1996).

Durch die Art der hier verfolgten Fragestellung wird nicht nur die (strukturbildende) $\mathrm{Be}$ deutung des geographischen Raumes für die handelnden Individuen explizit anerkannt (z.B.

1) Für kritische Anmerkungen und hilfreiche Hinweise danke ich Angelika Tölke, Gerda Neyer und Stephanie Stuck. Die multivariaten Modelle wurden während eines Gastaufenthaltes bei der SOEPGruppe am DIW Berlin geschätzt.

2) Leider erlauben es die hier zur Verfügung stehenden Daten nicht, alters- oder geschlechtsspezifische Heiratsraten auszuweisen. 
Esser 1988), sondern es wird insbesondere der von der einschlägigen Forschung bislang weitgehend vernachlässigten Rolle des Mannes als eigenständiges Subjekt im Prozeß der Familiengründung in angemessener Weise Rechnung getragen (z.B. Goldscheider/Kaufman 1996; Tölke/Hank 2004).

\section{Abbildung 1: Räumliche Verteilung durchschnittlicher roher Heiratsziffern in westdeutschen Stadt- und Landkreisen, 1986/93}

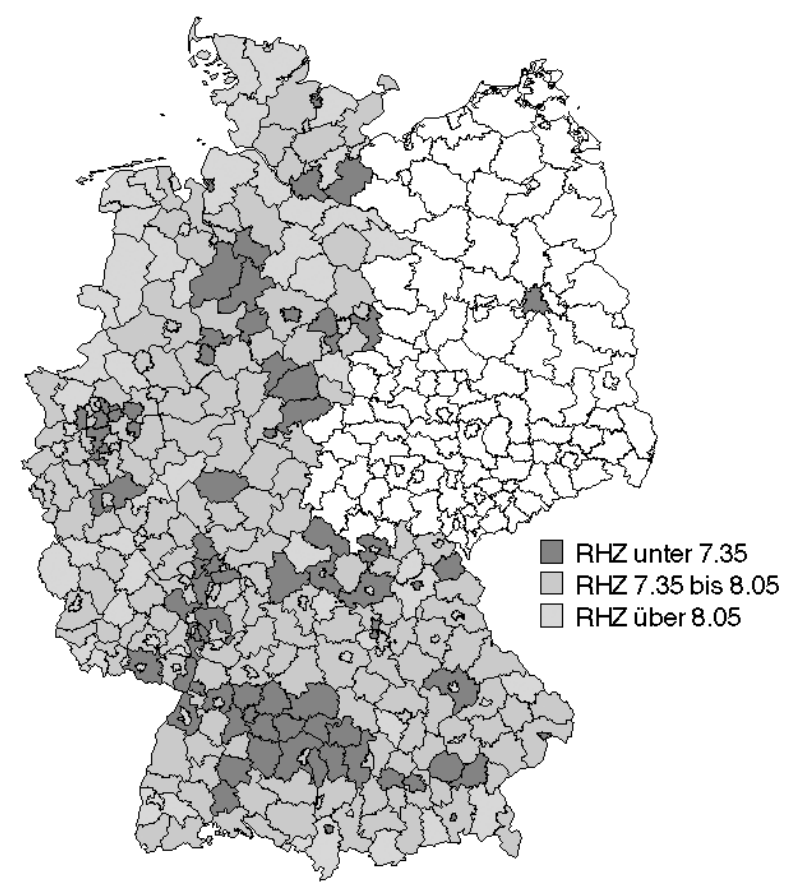

Anmerkungen:

Die mittlere Kategorie umfaßt die Kreise des zweiten und dritten Quartils der Häufigkeitsverteilung. Quelle: DJI Regionaldatenbank, eigene Darstellung.

\section{Heiratsentscheidungen aus regionaler Perspektive}

Wenn - wie in der westdeutschen Gesellschaft - Elternschaft üblicherweise in der Ehe stattfindet (z.B. Billari/Kohler 2002), gleichzeitig jedoch nicht-eheliche Partnerschaften eine sozial akzeptierte und kaum mehr benachteiligte Form des Zusammenlebens darstellen, gewinnt die Frage an Bedeutung, unter welchen Bedingungen Individuen heiraten (vgl. Waite et al. 2000).

Auf der individuellen Entscheidungsebene ist häufig argumentiert worden, dass die Heiratswahrscheinlichkeit insbesondere von Bildungsstand und sozio-ökonomischem Status, sowie vom familiären Hintergrund und Wertorientierungen abhängt (z.B. Clarkberg/Stolzenberg/Waite 1995; Cooney/Hogan 1991; Oppenheimer 2000). Für Westdeutschland konnte Huinink (1995, S.255ff.) u.a. nachweisen, dass Männer aus Herkunftsfamilien mit höherem sozialen Status später heiraten. Gleiches gilt für jene, die sich in Ausbildung befinden, wäh- 
rend erwerbstätige Männer - insbesondere solche, die in statushohen Berufen arbeiten - die höchsten Übergangsraten zur ersten Ehe aufweisen (vgl. auch Tölke 2004).

Mit Blick auf die kontextuellen Determinanten der Eheschließung hat sich die bisherige Forschung hauptsächlich mit der Rolle von Heiratsmärkten befaßt (z.B. Lichter/LeClere/ McLaughlin 1991), wo die Auswahl potenzieller Partnerinnen häufig in räumlich begrenzten sozialen Netzwerken erfolgt (South/Crowder 2000, S.1069; Lengerer 2001). Bei der Analyse lokaler Heiratsmärkte ist es sinnvoll, zwischen der >Quantität< und der >Qualität< des Pools möglicher Partnerinnen zu unterscheiden. Die Ergebnisse einer Studie von Lloyd und South (1996) zeigen, dass ein Überschuß an Frauen den Eintritt von Männern in den Ehestand fördert, was im Einklang mit Theorien zum Suchverhalten auf Heiratsmärkten steht (z.B. Oppenheimer 1988). Bei zusätzlicher Berücksichtigung der Rolle von Qualitätserwägungen für Heiratsentscheidungen wird erwartet, dass die individuelle Neigung zu heiraten mit der Zahl wirtschaftlich attraktiver Partnerinnen in einer Region steigt, da sich mit diesen die eigenen Ressourcen in vorteilhafter Weise poolen lassen (z.B. Lichter/LeClere/McLaughlin 1991). Eine höhere Bildungs- und Erwerbsbeteiligung von Frauen führt jedoch gleichzeitig zu einer größeren wirtschaftlichen Unabhängigkeit potenzieller Partnerinnen, was häufig als Ursache für eine Verzögerung von Eheschließungen betrachtet wird (vgl. hierzu auch die ausführliche Diskussion bei Oppenheimer [1997]).

Heiratsmärkte bilden jedoch nur einen Teil der weiter zu fassenden regionalen Opportunitätsstruktur (vgl. Hank 2002, Abschnitt 2.2). Unabhängig von der eigenen Einkommensoder Erwerbssituation kann beispielsweise auch die individuelle Wahrnehmung der allgemeinen wirtschaftlichen Lage die jeweilige Bereitschaft zur Familiengründung beeinflussen (z.B. Huinink 1995, S.251). Schlechte Arbeitsmarktperspektiven könnten den Eintritt in eine Ehe verzögern, da unsicher ist, inwieweit der Mann (gegenwärtig oder zukünftig) seiner traditionellen Rolle als Haupternährer der Familie nachkommen kann.

Der wahrscheinlich meistgenannte kontextuelle Einflußfaktor auf individuelles Verhalten ist der Urbanisierungsgrad einer Region (z.B. Huinink/Wagner 1989; Linde 1988, S.183ff.). Dabei wird angenommen, dass die räumlichen Kategorien $>$ Stadt $<$ und $>$ Land $<$ durch unterschiedliche sozio-kulturelle Milieus charakterisiert werden können, insbesondere mit Blick auf die in der jeweiligen Bevölkerung dominierenden familienbezogenen Einstellungen und Werte. Außerdem ist anzunehmen, dass soziale Kontrolle mit zunehmender Bevölkerungsgröße abnimmt, da ländliche Regionen im Allgemeinen durch eine stärkere Einbindung von Individuen in die örtliche Gemeinschaft gekennzeichnet sind (vgl. Linde 1988, S.184f.; South/Crowder 2000, S.1073).

Da sich die Fragestellung dieser Untersuchung nicht auf die Partnerschaftsgründung an sich, d.h. auf die Wahl einer bestimmten Partnerin, bezieht, sondern auf den Übergang zur ersten Ehe, d.h. auf die Wahl einer bestimmten Partnerschaftsform, könnten kollektive Verhaltenserwartungen der sozialen Umwelt möglicherweise von größerer Bedeutung für die individuelle Entscheidungsfindung sein, als strukturelle Merkmale des Heiratsmarktes. Regionale Unterschiede im Modernisierungs- und Individualisierungsgrad sind in diesem Zusammenhang häufig genannte mögliche Determinanten der räumlichen Verbreitung verschiedener Formen partnerschaftlichen Zusammenlebens (z.B. Klein 2000, S.61f.; Lengerer 1999). Obwohl man annehmen kann, dass sich normative Erwartungen bezüglich des Alters und der Reihenfolge, in denen bestimmte familiäre Ereignisse stattfinden sollten, je nach (sozialräumlichem) Kontext unterscheiden, sind sie empirisch nur schwer zu messen (z.B. Marini 1984). Die Berücksichtigung unbeobachteter regionaler Heterogenität in der multivariaten Analyse gewinnt hierdurch zusätzlich an Bedeutung. 


\section{Daten und Methode}

Datenbasis der Studie ist das Sozio-oekonomische Panel des DIW Berlin (vgl. SOEP-Gruppe 2001), eine in Westdeutschland seit 1984 jährlich durchgeführte Längsschnittuntersuchung privater Haushalte. Die vollständige Heiratsbiographie aller an der Befragung teilnehmenden Personen ist im Datensatz enthalten, wobei sich die vorliegende Analyse allein auf die erste Eheschließung in Westdeutschland lebender Männer bis zum Jahr 1999 bezieht.

Den Individualdaten des SOEP können Informationen über die Landkreise und kreisfreien Städte, in denen die Befragten leben, zugespielt werden. Die DJI Regionaldatenbank enthält Kreisindikatoren für die zweite Hälfte der 1980er Jahre, während entsprechende Informationen für die Mitte der 1990er Jahre in der Statistik regional Datenbank der Statistischen Ämter des Bundes und der Länder zu finden sind. Da die meisten interessierenden Regionalvariablen nur für jeweils einen Zeitpunkt in den 1980er und 1990er Jahren vorliegen, können zeitabhängige Kontextmerkmale ihre Ausprägung nicht jährlich ändern, sondern lediglich zwischen zwei als gleich lang definierten Zeiträumen, deren erster die Jahre 1984 bis 1991 umfaßt, während der zweite die Periode 1992 bis 1999 abdeckt.

Untersucht werden westdeutsche und ausländische Männer im Alter von 20 bis 40 Jahren. Personen, die während des Untersuchungszeitraums innerhalb Westdeutschlands umziehen, werden an ihren neuen Wohnort verfolgt. Damit besteht die hier verwendete Stichprobe aus 2.280 unverheirateten Männern in 300 Stadt- und Landkreisen. Da jedes Individuum mehrfach beobachtet werden kann, gehen insgesamt 15.223 Beobachtungen in die Analyse ein. Die Anzahl der Ereignisse (d.h. Erstheiraten) im Untersuchungszeitraum beträgt 850 . Weitere deskriptive Statistiken finden sich in Tabelle 1.

Folgende individuelle (d.h. personenbezogene) Kontrollvariablen werden in die Regression aufgenommen:

- das Alter und - da eine nicht-lineare Beziehung zur abhängigen Variablen angenommen wird - das quadrierte Alter der Befragten,

- zeitabhängige binäre Variablen, die den höchsten (beruflichen) Bildungsabschluss zum Zeitpunkt des jeweiligen Interviews angeben: in Ausbildung, kein Ausbildungsabschluss, beruflicher Ausbildungsabschluss (Referenzkategorie), Universitätsabschluss,

- eine zeitabhängige binäre Variable, die den Wert 1 annimmt, wenn der Befragte vollzeiterwerbstätig ist,

- sowie eine zeitunabhängige binäre Variable, die den Wert 1 annimmt, wenn der Befragte Ausländer ${ }^{3}$ ist.

Das Hauptaugenmerk gilt jedoch den Kontextmerkmalen auf der Kreisebene:

- der durchschnittliche Frauenanteil an der Bevölkerung im Alter von 20 bis 40 Jahren in der Periode 1995-1997 (in Prozent) (zeitunabhängig),

- die regionale Frauenerwerbsquote (in Prozent) (zeitabhängig, 1987/1995),

- eine zeitunabhängige binäre Variable, die den Wert 1 annimmt, wenn ein Kreis eine Bevölkerungsdichte von weniger als 150 Einwohnern pro km² aufweist (= ländlicher Raum),

- und die regionale Arbeitslosenquote (in Prozent) (zeitabhängig, 1987/1996).

3) Hierbei handelt es sich um Befragte der SOEP-Stichprobe B, also Griechen, Italiener, Spanier, Türken und Männer aus Ex-Jugoslawien, die bereits 1984 in der Bundesrepublik gelebt haben. 
Tabelle 1: Deskriptive Statistiken

\begin{tabular}{|l|c|}
\hline Variablen & Mittelwert (Standardabweichung) \\
\hline Individualmerkmale & $26.0(4.8)$ \\
Alter & $698.4(268.3)$ \\
Quadriertes Alter & .20 \\
In Ausbildung & .18 \\
Kein Ausbildungsabschluss & .52 \\
Beruflicher Ausbildungsabschluss & .09 \\
Universitätsabschluss & .59 \\
Vollzeit erwerbstätig & .23 \\
Ausländer & \\
Kontextmerkmale & $48.6(0.8)$ \\
Frauen-Anteil & $41.2(3.4)$ \\
Frauenerwerbsquote & .17 \\
Ländlicher Raum & $8.6(3.1)$ \\
Arbeitslosenquote & 850 \\
\hline N (Ereignisse) & 300 \\
N (Kreise) & 2,880 \\
N (Männer) & 15,223 \\
N (Beobachtungen) & \\
\hline
\end{tabular}

Anmerkungen:

Mittelwerte beziehen sich auf beobachtete Personenjahre.

Standardabweichungen für binäre Variablen werden nicht ausgewiesen.

Quelle: SOEP 1984-1999, DJI Regionaldatenbank, Statistik regional 1999, eigene Berechnungen.

Die Wahrscheinlichkeit, innerhalb eines Intervalls von einem Jahr zu heiraten, wird in dieser Untersuchung mit Hilfe zeitdiskreter logistischer Mehrebenenmodelle geschätzt (z.B. Barber et al. 2000; siehe auch Hank 2002, 2003). Bei dem von uns gewählten Verfahren kann die Regressionskonstante mit dem jeweiligen Kontext (hier: Kreis) variieren, d.h. sie besteht aus einer fixen und einer zufälligen Komponente ('random intercept model'; vgl. Snijders/Bosker 1999, Kapitel 4). Neben zeitabhängigen und zeitunabhängigen Individualvariablen sowie zeitkonstanten Kontextvariablen findet sich auf der rechten Seite der Regressionsgleichung ein normalverteilter regionaler Zufallskoeffizient $\left(\sigma_{u}\right)$. Er beschreibt die Abweichung jedes einzelnen Kontexts vom fixen Teil der Regressionskonstante. Damit wird nicht nur für die Korrelation zwischen Individuen innerhalb desselben Kontexts kontrolliert, sondern es werden auch sonst unbeobachtete regionale Effekte erfasst. Unterscheidet sich $\sigma_{u}$ statistisch signifikant von 0 , liegt ein solcher latenter Kontexteffekt vor.

\section{Ergebnisse der multivariaten Analyse}

Um den Übergang in die erste Ehe zu analysieren werden zwei Modelle geschätzt. Während in Modell 1 nur für individuelle Merkmale der Männer kontrolliert wird, werden in Modell 2 zusätzlich die oben genannten Kontextvariablen aufgenommen. Beide Modelle berücksichtigen unbeobachtete Heterogenität auf der Regionalebene. Die Regressionsergebnisse sind in Tabelle 2 dargestellt. 
Hinsichtlich Vorzeichen und statistischer Signifikanz ergeben sich für die Koeffizienten der individuellen Kontrollvariablen die erwarteten Resultate (Modell 1). Der Alterseffekt wirkt nicht-monoton, d.h. die Heiratsneigung eines Mannes steigt zunächst mit zunehmendem Alter, sinkt jedoch wieder in späteren Lebensphasen. Dies stimmt gut mit neueren Befunden aus dem DJI-Familiensurvey überein, die auf eine starke Altersabhängigkeit von Erstheirat und erster Elternschaft auch bei Männern hindeuten (vgl. Tölke 2004). Bei den Ausbildungs- und Erwerbsvariablen zeigt sich, dass Männer in Ausbildung ebenso wie jene ohne Ausbildungsabschluss eine geringere Ehewahrscheinlichkeit aufweisen, während solche, die über einen Universitätsabschluss verfügen oder vollzeiterwerbstätig sind, eher heiraten, als Männer in der jeweiligen Referenzkategorie. Außerdem ist die Wahrscheinlichkeit eine Ehe einzugehen bei Ausländern höher als bei westdeutschen Männern.

In Modell 2 bleiben die Koeffizienten der Individualvariablen nach Aufnahme der Kontextindikatoren unverändert. Ebensowenig verringert sich der in beiden Modellen hochsignifikante regionale Zufallseffekt $\left(\sigma_{u}\right)$. Die Regionalvariablen tragen damit weder zur Erklärung der Varianz zwischen den Kreisen bei, noch verbessern sie in ihrer Summe die Güte des Modells. Dennoch lässt sich ein statistisch signifikanter Einfluß der Frauenerwerbsquote feststellen: eine höhere Erwerbsbeteiligung von Frauen auf dem regionalen Arbeitsmarkt verringert die Wahrscheinlichkeit, daß ein Mann im Beobachtungszeitraum eine erste Ehe eingeht. ${ }^{4}$

Tabelle 2: Ergebnisse der zeitdiskreten logistischen Mehrebenenmodelle

\begin{tabular}{|l|c|c|c|c|c|c|}
\hline \multicolumn{4}{|c|}{ Modell 1 } & \multicolumn{2}{c|}{ Modell 2 } \\
\hline & $\beta$ & s.e. & Sig. & $\beta$ & s.e. & Sig. \\
\hline Alter & .79 & .09 & $* * *$ & .81 & .09 & $* * *$ \\
\hline Quadriertes Alter & -.01 & .00 & $* * *$ & -.01 & .00 & $* * *$ \\
\hline In Ausbildung & -.35 & .16 & $* *$ & -.34 & .16 & $* *$ \\
\hline Kein Ausbildungsabschluss & -.20 & .11 & $*$ & -.20 & .11 & $*$ \\
\hline Universitätsabschluss & .24 & .11 & $* *$ & .26 & .11 & $* *$ \\
\hline Vollzeit erwerbstätig & .78 & .11 & $* * *$ & .77 & .11 & $* * *$ \\
\hline Ausländer & .38 & .09 & $* * *$ & .38 & .09 & $* * *$ \\
\hline Frauen-Anteil & - & & & -.06 & .05 & \\
\hline Frauenerwerbsquote & - & & & -.03 & .01 & $* *$ \\
\hline Ländlicher Raum & - & & & .03 & .11 & \\
\hline Arbeitslosenquote & - & & & -.02 & .01 & \\
\hline Konstante & -14.93 & 1.27 & $* * *$ & -13.91 & 1.33 & $* * *$ \\
\hline$\sigma_{u}$ & .22 & .07 & $* *$ & .22 & .07 & $* *$ \\
\hline-2 Log likelihood & \multicolumn{2}{|l|}{6,206} & & & 6.196 & \\
\hline
\end{tabular}

Anmerkungen:

Referenzkategorie für die Bildungsvariablen ist >beruflicher Ausbildungsabschluss $<$.

Signifikanz: $*<.10 ; * *<.05 ; * * * .01$.

Quelle: SOEP 1984-1999, DJI Regionaldatenbank, Statistik regional 1999, eigene Berechnungen.

\section{Diskussion}

Im vorliegenden Beitrag wird aus einer Mehrebenenperspektive heraus das Heiratsverhalten westdeutscher Männer untersucht. Die multivariate Analyse bestätigt zunächst den aus frü-

4) Zu einem ähnlichen Ergebnis kommen auch Lloyd und South (1996, S.1114) bei einer Analyse USamerikanischer Daten. 
heren Studien bereits bekannten starken Zusammenhang zwischen der wirtschaftlichen Stellung eines Mannes und der Heiratsentscheidung (z.B. Oppenheimer 2000). Gut ausgebildete und vollerwerbstätige Männer weisen demnach die höchste Wahrscheinlichkeit einer Eheschließung auf. Im Gegensatz zur individuellen Erwerbssituation spielt die Lage auf dem regionalen Arbeitsmarkt - soweit sie über die jeweilige Arbeitslosenquote gemessen wird keine signifikante Rolle. Lebt ein Mann jedoch in einem Stadt- oder Landkreis mit einer hohen Frauenerwerbsquote, sinkt die Heiratsneigung.

Diese negative Beziehung zwischen der Erwerbsbeteiligung von Frauen auf dem regionalen Arbeitsmarkt und dem Übergang zur ersten Ehe bei Männern könnte als Bestätigung von Hypothesen gewertet werden, die in der ökonomischen Unabhängigkeit von Frauen auf dem lokalen Heiratsmarkt eine der Hauptursachen für den Aufschub, bzw. das Ausbleiben von Eheschließungen sehen. Dieser zunächst plausibel erscheinende Erklärungsansatz erweist sich jedoch bei genauerer Betrachtung als nicht unproblematisch. In einem kritischen Literaturüberblick weist z.B. Oppenheimer (1997, S.449) darauf hin, dass die sogenannte »Unabhängigkeithypothese « nur durch solche Studien empirisch gestützt wird, die auf Aggregatdaten basieren. Untersuchungen auf der Grundlage von Individualdaten zeigen hingegen im Allgemeinen, dass eine höhere Bildung, Erwerbstätigkeit und das Einkommen von Frauen keinen, oder sogar einen tendenziell positiven Effekt auf die Heiratsneigung haben. In Übereinstimmung hiermit findet Hank (2003), dass Frauen ohne Ausbildungsabschluß niedrigere Übergangsraten zur ersten Ehe aufweisen als jene mit Berufsausbildung; ein Einfluß der Frauenerwerbsquote kann nicht nachgewiesen werden.

Es erscheint daher als sinnvoll, eine komplementäre Interpretation der Beziehung zwischen (aggregierter) Frauenerwerbstätigkeit und (individuellem) Heiratsverhalten von Männern in Betracht zu ziehen. Eine hohe Erwerbsbeteiligung von Frauen könnte z.B. nicht nur auf einen gesellschaftlichen Zusammenhang hinweisen, in dem diese in geringerem Maße ökonomisch von Männern abhängig sind, sondern sie könnte ebenso einen sozialräumlichen Kontext markieren, in dem gleichberechtigte Geschlechterrollen sowie die individuelle $\mathrm{Au}-$ tonomie (auch der Männer!) gegenüber äußeren institutionellen Autoritäten verhältnismäßig stark ausgeprägt sind (vgl. Lesthaeghe/Neels 2002; Sackmann/Häussermann 1994).

Folgt man diesem Ansatz, lässt sich - soweit es um das Wirken kontextueller Faktoren geht - die zwischen den westdeutschen Stadt- und Landkreisen beobachtete Heterogenität im Heiratsverhalten wesentlich auf regional unterschiedliche Normen, Einstellungen und Werte zurückführen. Diese konstituieren, unabhängig von individuellen Handlungsentscheidungen, raumgebundene sozio-kulturelle Milieus mit spezifischen partnerschafts- und familienbezogenen kollektiven Verhaltenserwartungen und Wertorientierungen. Nauck (1995, S.96; Hervorhebung im Original) spricht in diesem Zusammenhang von einem »Mechanismus der kulturellen Tradierung und Perpetuierung von normativen Mustern der Lebensführung [der] auch dann wirkt, wenn soziale Kontrolle nur minimal ausgeübt wird. Dieser Mechanismus beschreibt noch am genauesten, was mit der Verhaltenswirksamkeit sozial-kultureller $>$ Milieus $<$ bezeichnet wird. « Deren Existenz wird indirekt auch durch den statistisch signifikanten latenten Kontexteffekt auf die Wahrscheinlichkeit einer Eheschließung belegt, der bereits bei der Untersuchung der Familiengründung von Frauen nachgewiesen werden konnte (vgl. Hank 2003).

Die räumliche Dimension dieser Milieus entspricht jedoch nicht einer einfachen StadtLand-Dichotomie: der entsprechende Indikator hat in unserem Modell keine nennenswerte Erklärungskraft. Letztlich dürften es in erster Linie >natürliche Nachbarschaften< sein, die den Raum für soziale Identifikationsprozesse bilden und die Entwicklung von regionalen Mentalitäten prägen (Kopp 2000, S.113). In einer qualitativen Untersuchung können z.B. Helfferich et al. (2004) zeigen, dass sowohl im dörflichen Freiburger Umland als auch im bis 
heute durch den Bergbau geprägten Milieu Gelsenkirchens die soziale Einbindung des Einzelnen in erster Linie über ein dichtes Kontaktgeflecht mit Freunden, Nachbarn, Eltern, Bekannten, die >man kennt< oder >trifft $<$, erfolgt. Diese kollektive Eingebundenheit beeinflußt neben individualbiografischen Eigenheiten - die Entwicklung der Biografiekonzepte der dort lebenden Männer wesentlich mit, etwa im Hinblick auf die Frage, wann der >richtige< Zeitpunkt für eine Eheschließung bzw. Familiengründung (und damit der »Transformation« der eigenen Männlichkeit) gekommen ist.

Insbesondere vor dem Hintergrund der Feststellung von Klein (2003, S.525), »dass Heiratsmarkt und Beziehungsstabilität zu den bislang vernachlässigten Einflussgrößen des generativen Verhaltens gehören«, ergeben sich aus den Ergebnissen der vorliegenden Untersuchung zwei Konsequenzen. Erstens darf die Bedeutung von Heiratsmärkten nicht auf die Qualität und Quantität erreichbarer PartnerInnen reduziert werden, denn sie sind gleichzeitig einflussreiche »Orte sozialer Kontrolle oder [...] der Tradierung von Lebensformen und kulturellen Mustern « (Nauck 1995, S.95). ${ }^{5}$ Dies gilt in gleichem Maße für Männer und Frauen, d.h. beide Partner bringen nicht nur ihre individuellen Erfahrungen und Erwartungen, sondern auch ihre jeweiligen regionalen Prägungen in die Beziehung oder in die Familie mit ein. Daher erscheint, zweitens, eine Paarperspektive innerhalb eines $>$ Drei-Ebenen-Modells $<$ (Individuum-Paarbeziehung-Region) zur Erklärung familialer Prozesse nicht nur vielversprechend, sondern nahezu unabdingbar.

\section{Literatur}

Barber, J.S./ Murphy, S.A./ Axinn, W.G./ Marples, J. (2000): Discrete-time multilevel hazard analysis, in: M.E. Sobel/ M.P. Becker (Hrsg.), Sociological Methodology 30, Washington: Blackwell Publishers, S.201-235.

Bertram, H. (1993): Was bietet der Regionalansatz für Wissenschaft, Politik und Praxis?, in: Diskurs 1/ 93, S.37-42.

Bertram, H./ Nauck, B./ Klein, T. (Hrsg.) (2000): Solidarität, Lebensformen und regionale Entwicklung, Opladen: Leske + Budrich.

Billari, F./ Kohler, H.-P. (2002): The impact of union formation dynamics on first births in West Germany and Italy: Are there signs of convergence?, in: E. Klijzing/ M. Corijn (Hrsg.), Dynamics of Fertility and Partnership in Europe: Insights and Lessons from Comparative Research (Bd. 2), Genf/ New York: United Nations, S.43-58.

Clarkberg, M./ Stolzenberg, R.M./ Waite, L.J. (1995): Attitudes, Values, and Entrance into Cohabitational versus Marital Unions, in: Social Forces 74, S.609-634.

Cooney, T.M./ Hogan, D.P. (1991): Marriage in an Institutionalized Life Course: First Marriage among American Men in the Twentieth Century, in: Journal of Marriage and the Family 53, S.178-190.

Esser, H. (1988): Sozialökologische Stadtforschung und Mehr-Ebenen-Analyse, in: J. Friedrichs (Hrsg.), Soziologische Stadtforschung (Kölner Zeitschrift für Soziologie und Sozialpsychologie, Sonderheft 29), S.33-55.

Goldscheider, F.K./ Kaufman, G. (1996): Fertility and Commitment: Bringing Men Back In, in: Population and Development Review 22 (Supplement), S.87-99.

Hank, K. (2002): Regional Social Contexts and Individual Fertility Decisions: A Multilevel Analysis of First and Second Births in Western Germany, in: European Journal of Population 18, S.281-299.

Hank, K. (2003): Eine Mehrebenenanalyse der Familiengründung westdeutscher Frauen in den Jahren 1984 bis 1999, in: Kölner Zeitschrift für Soziologie und Sozialpsychologie 55, S.79-98.

5) Dabei ist nicht notwendigerweise von einer strengen räumlichen Identität zwischen Heiratsmärkten und >Orten sozialer Kontrolle` auszugehen. 
Helfferich, C./ Klindworth, H./ Krumm, S./ Walter, W. (2004): Familienentwicklung als Transformation von Männlichkeit, in: A. Tölke/ K. Hank (Hrsg.), Männer - Das >vernachlässigte< Geschlecht in der Familienforschung (Sonderheft 4 der Zeitschrift für Familienforschung), Wiesbaden: VS Verlag für Sozialwissenschaften, im Druck.

Huinink, J. (1995): Education, Work, and Family Patterns of Men: The Case of West Germany., in: H.-P. Blossfeld (Hrsg.), The new role of women: Family formation in modern societies, Boulder: Westview Press, S.247-262.

Huinink, J./ Wagner, M. (1989): Regionale Lebensbedingungen, Migration und Familienbildung, in: Kölner Zeitschrift für Soziologie und Sozialpsychologie 41, S.669-689.

Klein, T. (2000): Partnerwahl und Partnerschaftsformen im regionalen Kontext, in: H. Bertram/ B. Nauck/ T. Klein (Hrsg.), Solidarität, Lebensformen und regionale Entwicklung, Opladen: Leske + Budrich, S.59-82.

Klein, T. (2003): Die Geburt von Kindern in paarbezogener Perspektive, in: Zeitschrift für Soziologie 32, S.506-527.

Kopp, J. (2000): Geburtenentwicklung in Ost- und Westdeutschland, in: H. Bertram/ B. Nauck/ T. Klein (Hrsg.), Solidarität, Lebensformen und regionale Entwicklung, Opladen: Leske + Budrich, S.83-135.

Lengerer, A. (1999): Regionale Disparitäten der Verbreitung und Entwicklung Nichtehelicher Lebensgemeinschaften, in: T. Klein/ W. Lauterbach (Hrsg.), Nichteheliche Lebensgemeinschaften, Opladen: Leske + Budrich, S.139-166.

Lengerer, A. (2001): Wo die Liebe hinfällt - ein Beitrag zur >Geographie< der Partnerwahl, in: T. Klein (Hrsg.), Partnerwahl und Heiratsmuster. Sozialstrukturelle Voraussetzungen der Liebe, Opladen: Leske + Budrich, S.133-162.

Lesthaeghe, R./ Neels, K. (2002): From the First to the Second Demographic Transition: An Interpretation of the Spatial Continuity of Demographic Innovation in France, Belgium and Switzerland, in: European Journal of Population 18, S.325-360.

Lichter, D.T./ LeClere, F.B./ McLaughlin, D.K. (1991): Local Marriage Markets and the Marital Behavior of Black and White Women, in: American Journal of Sociology 96, S.843-867.

Linde, H. (1988): Das sozialökologische Gefälle der ehelichen Fruchtbarkeit im Prozeß der Nachwuchsbeschränkung in Deutschland, in: C. Höhn et al. (Hrsg.), Demographie in der Bundesrepublik Deutschland: vier Jahrzehnte Statistik, Forschung und Politikberatung. Festschrift für Karl Schwarz, Boppard am Rhein: Boldt, S. 169-186.

Lloyd, K.M./ South, S.J. (1996): Contextual Influences on Young Men's Transition to First Marriage. in: Social Forces 74, S.1097-1119.

Marini, M.M. (1984): Age and Sequencing Norms in the Transition to Adulthood, in: Social Forces 63, S.229-244.

Nauck, B. (1995): Regionale Millieus von Familien in Deutschland nach der politischen Vereinigung, in: B. Nauck/ C. Onnen-Isemann (Hrsg.), Familie im Brennpunkt von Wissenschaft und Forschung, Neuwied: Luchterhand, S.91-121.

Nauck, B./ Bertram, H. (Hrsg) (1995): Kinder in Deutschland: Lebensverhältnisse von Kindern im Regionalvergleich, Opladen: Leske + Budrich.

Oppenheimer, V.K. (1988): A Theory of Marriage Timing, in: American Journal of Sociology 94, S.563591.

Oppenheimer, V.K. (1997): Women's Employment and the Gain to Marriage: The Specialization and Trading Model, in: Annual Review of Sociology 23, S.431-453.

Oppenheimer, V.K. (2000): The Continuing Importance of Men's Economic Position in Marriage Formation, in: L.J. Waite et al. (Hrsg.), The Ties That Bind. Perspectives on Marriage and Cohabitation, New York: Aldine de Gruyter, S.283-301.

Sackmann, R./ Häussermann, H. (1994): Do regions matter? Regional differences in female labour-market participation, in: Environment and Planning (A) 26, S.1377-1396. 
Snijders, T.A.B./ Bosker, R.J. (1999): Multilevel analysis: An introduction to basic and advanced multilevel modeling, London: Sage.

SOEP-Gruppe (2001): The German Socio-Economic Panel (GSOEP) after more than 15 years - Overview, in: E. Holst et al. (Hrsg.), Proceedings of the 2000 Fourth International Conference of German Socio-Economic Panel Study Users (GSOEP2000), Vierteljahrshefte zur Wirtschaftsforschung 70, S.7-14.

South, S.J./ Crowder, K.D. (2000): The Declining Significance of Neighborhoods? Marital Transitions in Community Context., in: Social Forces 78, S.1067-1099.

Tölke, A. (2004): Die Bedeutung von Herkunftsfamilie, Berufsbiografie und Partnerschaften für den Übergang zur Ehe und Vaterschaft, in: A. Tölke/ K. Hank (Hrsg.), Männer - Das > vernachlässigte< Geschlecht in der Familienforschung (Sonderheft 4 der Zeitschrift für Familienforschung), Wiesbaden: VS Verlag für Sozialwissenschaften, im Druck.

Tölke, A./ K. Hank (Hrsg.) (2004): Männer - Das > vernachlässigte< Geschlecht in der Familienforschung (Sonderheft 4 der Zeitschrift für Familienforschung), Wiesbaden: VS Verlag für Sozialwissenschaften, im Druck.

Waite, L.J., et al. (Hrsg.) (2000): The Ties That Bind. Perspectives on Marriage and Cohabitation, New York: Aldine de Gruyter.

Dr. Karsten Hank

Mannheimer Forschungsinstitut Ökonomie und demographischer Wandel (MEA)

Universität Mannheim L 13, 17

68131 Mannheim

hank@mea.uni-mannheim.de 